Vo, D.-B. and Brewster, S. (2020) Investigating the Effect of Tactile Input and Output Locations for Drivers' Hands on In-Car Tasks Performance. In: 12th International Conference on Automotive User Interfaces and Interactive Vehicular Applications (AutomotiveUI '20), 21-22 Sep 2020, ISBN 9781450380652 (doi:10.1145/3409120.3410656)

There may be differences between this version and the published version. You are advised to consult the publisher's version if you wish to cite from it.

(C) The Authors 2020. This is the author's version of the work. It is posted here for your personal use. Not for redistribution. The definitive Version of Record was published in the Proceedings of the 12th International Conference on Automotive User Interfaces and Interactive Vehicular Applications (AutomotiveUI '20), 21-22 Sep 2020, ISBN 9781450380652 (doi:10.1145/3409120.3410656)

\title{
http://eprints.gla.ac.uk/222486/
}

Deposited on: 09 September 2020

Enlighten - Research publications by members of the University of 


\section{Investigating the effect of tactile input and output locations for drivers' hands on in-car tasks performance}

\author{
Dong-Bach Vo \\ dong-bach.vo@glasgow.ac.uk \\ University of Glasgow \\ Glasgow, United Kingdom
}

\author{
Stephen A. Brewster \\ stephen.brewster@glasgow.ac.uk \\ University of Glasgow \\ Glasgow, United Kingdom
}
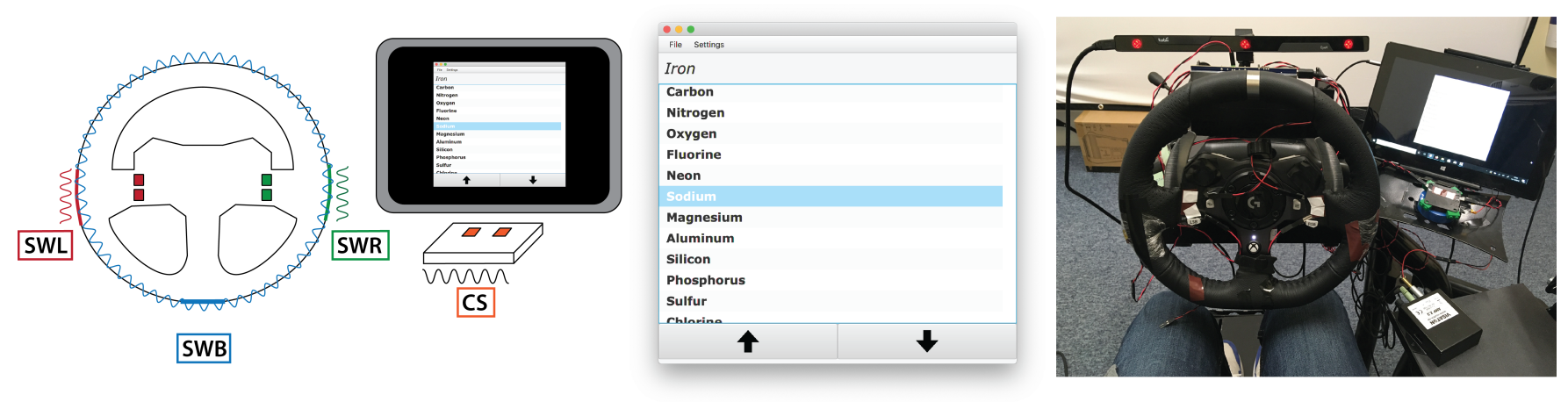

Figure 1: Steering wheel and centre console prototypes used in the study. Tactile switches (the coloured squares) for controlling the scrolling list (the secondary task) are located on the left (SWL) and the right (SWR) side of the steering wheel, and on the centre console (CS). Tactile feedback can be felt on the left, the right and on both sides (SWB) of the steering wheel, and on the centre console.

\begin{abstract}
This paper reports a study investigating the effects of tactile input and output from the steering wheel and the centre console on non-driving task performance. While driving, participants were asked to perform list selection tasks using tactile switches and to experience tactile feedback on either the non-dominant, dominant or both hands as they were browsing the list. Our results show the average duration for selecting an item is $30 \%$ shorter when interacting with the steering wheel. They also show a $20 \%$ increase in performance when tactile feedback is provided. Our findings reveal that input prevails over output location when designing interaction for drivers. However, tactile feedback on the steering wheel is beneficial when provided at the same location as the input or to both hands. The results will help designers understand the trade-offs of using different interaction locations in the car.
\end{abstract}

\section{CCS CONCEPTS}

- Human-centered computing $\rightarrow$ User studies; Interaction techniques; Haptic devices.

\section{KEYWORDS}

tactile feedback, tactile input, steering wheel, interaction techniques, automotive user interaction, driver behavior, haptics, vehicle drivers, ground vehicle standard J287_201603

Conference'17, July 2017, Washington, DC, USA

2020. ACM ISBN 978-x-xxxx-xxxx-x/YY/MM...\$15.00

https://doi.org/10.1145/nnnnnnn.nnnnnnn
ACM Reference Format:

Dong-Bach Vo and Stephen A. Brewster. 2020. Investigating the effect of tactile input and output locations for drivers' hands on in-car tasks performance. In Proceedings of ACM Conference (Conference'17). ACM, New York, NY, USA, 8 pages. https://doi.org/10.1145/nnnnnnn.nnnnnnn

\section{INTRODUCTION}

With the growing sophistication of in-vehicle information systems (IVIS), drivers may be distracted from operating their cars safely [5]. However, tactile feedback from different locations in the vehicle can improve driving performance and safety [12, 27]. For instance, it can reduce the duration of glances off the road, minimising visual distraction [24]. Tactile feedback in cars can also improve driver experience. For example, separating tactile input and output can offer a variety of opportunities such as stimulating different body areas simultaneously to reinforce or refine tactile messages, enabling feedback from any type of surface and even for conveying information before and after the input action has taken place [31].

The hands are one of the most sensitive and pleasant areas of the body for experiencing tactile stimulation [1]. To deliver tactile feedback to drivers' hands, pressure of the skin against an actuated surface is required. Previous research has shown that maximising the contact area of the skin with the actuated surface increases sensitivity to vibratory stimuli [22]. However, research has found that providing tactile information to both hands simultaneously does not improve tactile precision [34]. Furthermore, there is evidence of a handedness effect for tactile perception; the left hand being generally more sensitive for right handed participants [34]. This suggests that the brain does not combine all haptic information 
equally but is biased towards one input with bimanual haptics [34] Other findings suggest that the brain processes tactile and spatial information from both hands as if they were coming from a single entity, resulting in one central representation [9]. In the light of this, further research is needed to understand how it affects driving performance and how to improve interaction with in-car systems. Moreover, no research has studied the effects of distal tactile feedback [19] on driving performance.

In this paper, we investigate the effects of separating tactile input and output on driving performance using the steering wheel and the centre console, as they are promising locations to deliver tactile feedback to the hands $[6,23]$. We report a study where participants were asked to drive and select an item in a scrolling list displayed on the centre console, using switches located on either the steering wheel or the centre console. While browsing the list, they experienced tactile feedback from either the steering wheel or the centre console. The results show that the performance of interacting using tactile switches located on the left side of steering wheel and experiencing tactile feedback on both hands was $30 \%$ faster than interacting with switches on the centre console while receiving tactile feedback on the non-dominant hand on the steering wheel. In addition, the performance of selecting an item in the list by interacting with tactile switches on the steering wheel improved by more than $20 \%$ when tactile feedback was provided.

\section{RELATED WORK}

One of the main benefits of using haptic feedback in the car is the capability to deliver information rapidly to drivers while ensuring safe driving. Therefore, this has received a lot of attention and significant efforts have been spent on surveying previous research for automatic driving control handover [28], improving driving safety [12], and more specifically, for warning drivers [21] from the steering wheel [4]. As most of the time in contact with the steering wheel and one of the most sensitive areas for perceiving tactile stimuli, the main research efforts have focused on designing vibrotactile feedback techniques either to warn or to guide drivers through their hands [1,27].

Tactile feedback can support input from the steering wheel. To preserve the mechanical switch properties on steering wheels equipped with tactile surfaces, Diwischek and Lisseman have optimised the haptic signal characteristics to improve the feedback of touch buttons [7]. Their results show that participants favour pure sine signals of $230 \mathrm{~Hz}$ over more complex signals. Other research has proposed new techniques for interacting with IVIS through the steering wheel $[2,13,39,41]$ but without the use of haptics, which may also give additional benefits. In a comparative study of pressure-based input on the centre console, $\mathrm{Ng}$ et al. found that item selection performance in large lists was improved with vibrotactile feedback while limiting visual distraction [23]. Their study showed that participants' input time was significantly shorter when vibrotactile feedback was given for browsing the list and validating the selection. The authors also reported that it gave more confidence to participants as they did not have to rely on visual feedback alone to assess the state of the IVIS. However, the feedback was not delivered from the steering wheel but from the centre console where the display screen and the input sensors were located. It is worth noting that, while some research has compared input techniques on the steering wheel and on the centre console [8], no research has ever compared the effect of tactile feedback experienced from the steering wheel and from the centre console yet.

While the advantage of direct feedback at the location of the interaction has been mentioned before [7], it is worth noting that separating input and tactile feedback in a driving context has been studied [32]. Distal tactile feedback can reinforce or refine haptic messages, enable feedback from any location and any type of surface, and even convey information before and after the input action has taken place [31]. Studies have demonstrated the benefits of distal tactile feedback $[10,15,19,20]$, but not in the automotive domain. For example, McAdam and Brewster have shown that such feedback can increase typing speed with soft keyboards on interactive surfaces [19]. They also found that it can improve target selection performance of items arranged around a dial [20]. Along the same lines, Henderson et al. found that distal vibrotactile feedback from a smart watch worn on the wrist resulted in better performance than without feedback in a Fitts' target selection task [15]. Therefore, the separation of input from tactile feedback has many potential benefits which could also apply in driving.

Tactile feedback in car can be cognitively demanding. In a comparative study of tactile techniques for menu control in cars, Grane et al. found that tactile feedback reduced lane deviation when drivers were busy with a secondary task [14]. However, they also found that the participants experienced difficulties to select an item in the menu with haptic feedback during the driving task, missing more lane changing signs when experiencing the tactile feedback alone. Additionally in [23], a participant reported that "vibrotactile feedback would allow them to count the number of items in the list without having to take their eyes off the driving task", possibly contributing to the increase of the cognitive demand to complete the task. Even though dealing with tactile stimuli in driving situations may be cognitively demanding, it may be possible to limit the demand. Prior research has found a handedness effect to haptic perception $[34,35]$. For example, in a study on the haptic perception of curved objects through the handles of a manipulandum, Squeri et al. showed that the non-dominant hand was more sensitive than the dominant in right-handed users [34]. Their data also suggest that redundant information on both hands did not improve perception. However, when separating input movement and tactile ouput across both index fingers for identifying the orientation of a triangle, Dupin et al. discovered that information is processed as if coming from the same hand, even when participants used one hand to move a slider and the other to feel a dynamic tactile stimulus [9]. The authors also suggest that movement of the hand could affect the sensory signal even when the the tactile signal is felt by the other hand. Applying this novel research to tactile feedback in car could reduce drivers' cognitive demand when interacting with the IVIS, which must be done with one hand while the other is on the wheel.

Because drivers are manipulating the steering wheel and interacting with the IVIS at the same time, it is unclear how tactile stimuli experienced through the hands affect driving performance. On one hand, stimulating both hands at the same time could be more demanding for no significant performance benefit [34]. On the other, simulating just a single hand raises even more questions. 
Since there is evidence of handedness in haptic perception, stimulating the most sensitive hand could save drivers' cognitive resources and optimise the haptic signal delivery [34]. Additionally, because the tactile and kinaesthetic signals are merged as if they originate from the same entity, even when separated across hands, distal tactile feedback could open new opportunities for designing better interaction techniques to keep drivers' sight on the road [9]. No research has investigated the effect of the separation of input and tactile output on task performance in car, and furthermore, no study has compared the performance of interaction on the steering wheel and the centre console.

In this paper, we are interested in understanding how tactile input and feedback can affect driving and non-driving task performance. We first introduce a bi-manual tactile design space for the steering wheel and centre console that takes into account the recent findings in haptic perception [9, 34]. We then report a user study which examines the performance of driving and selecting an item in a list displayed on the centre console while participants interact and experience tactile feedback on their hands at different locations on the steering wheel and on the centre console.

\section{A TACTILE INPUT AND OUTPUT DESIGN SPACE FOR THE CAR}

To investigate the effects of the location of tactile input and output on IVIS control and driving performance, a design space reflecting the possible ways to stimulate hands in a driving situation was defined. Three different locations easily reachable by the driver's hands were selected: the centre console touchscreen surface $(C S)$, and the left $(S W L)$ and right side $(S W R)$ of the steering wheel. To be able to compare the effects of tactile feedback when it is distant and co-located from input, we added touch switches on each side of the steering wheel and to the centre console. In our experimental setup, delivering vibrotactile feedback on the centre console meant the index finger of the dominant hand was stimulated (driving on the right side of the road). Vibration motors on the left and right of the steering wheel provided localised feedback to each palm. We were also interested in understanding the effect of redundant tactile output, i.e when both hands were stimulated simultaneously. Therefore, we introduced two additional output combinations to stimulate both hands at the same time: tactile output on the both sides of the steering wheel $(S W B)$, tactile output on left side of the steering wheel and on the centre console (CSWL) and tactile output on the right side of the steering wheel and on the centre console (CSWR). A total of 18 combinations of input and output were possible.

Out of the 18 possible combinations, only 9 were evaluated. Some were discarded due to interaction restrictions. First, when drivers interact with the centre console, the right hand and both hands cannot be stimulated from the steering wheel as they drive on the right side of the road. Therefore, $C S \times S W R$ and $C S \times S W B$ were dropped. Secondly, when drivers interact on the steering wheel, feedback from the centre console will not be perceived. As a result, 7 combinations with input from the steering wheel and tactile output from the centre console were dismissed. The 9 remaining combinations were $C S \times S W L, S W L \times S W R, S W R \times S W L$, $C S \times C S, S W L \times S W L, S W R \times S W R, C S \times C S W L, S W L \times S W B$, and finally

\begin{tabular}{ccccccccc}
\multicolumn{10}{c}{ Output } \\
\cline { 3 - 9 } & & $S W L$ & $S W R$ & $S W B$ & $C S$ & $C S W L$ & $C S W R$ & None \\
\cline { 2 - 9 } & $S W L$ & $\mathrm{X}$ & $\mathrm{X}$ & $\mathrm{X}$ & $\mathrm{D}$ & $\mathrm{D}$ & $\mathrm{D}$ & $\mathrm{B}$ \\
$\vec{\Xi}$ & $S W R$ & $\mathrm{X}$ & $\mathrm{X}$ & $\mathrm{X}$ & $\mathrm{D}$ & $\mathrm{D}$ & $\mathrm{D}$ & $\mathrm{B}$ \\
& $C S$ & $\mathrm{X}$ & $\mathrm{D}$ & $\mathrm{D}$ & $\mathrm{X}$ & $\mathrm{X}$ & $\mathrm{D}$ & $\mathrm{B}$
\end{tabular}

Table 1: Experimental design space: list of input and output locations. $\mathrm{X}$ denotes selected, $\mathrm{B}$ baseline, and $\mathrm{D}$ discarded combinations.

$S W R \times S W B$. To build a performance baseline, we also included 3 final combinations where no tactile feedback was provided during the interaction: $C S \times$ None, $S W L \times$ None, $S W R \times$ None. The combinations are summarised in Table 1.

From these combinations, we formulated 3 hypotheses:

- Combinations with no tactile feedback will yield worse performance than combinations with tactile feedback (H1);

- Combinations on the steering wheel will yield better performance than combinations on the centre console (H2);

- Combinations with tactile feedback on the non-dominant hand will perform better than combinations with tactile feedback on both hands (H3).

\section{STUDY}

A study was designed to test the effects of tactile input and output location on performance while driving. The study consisted of selecting an item in a scrolling list displayed on a screen located on the centre console $[23,24]$ using tactile input and output at different locations reachable by hands while driving.

\subsection{Experimental Design}

The experimental conditions were ordered following a Latin square design. For each combination, we created a list of 10 targets taken randomly from the list. In total, 10 (selections $) \times 12($ combinations $) \times$ $12($ participants $)=1440$ selections were performed in this withinsubjects study.

To compare the different combinations of input and output location, the performance of both primary and secondary tasks was measured. Driving performance was measured through lane deviation from the centre of the middle lane, which was calculated with the root mean square error. For the item selection task, the selection time (the time between the trial start and the selection of an item in the list) and target selection accuracy were measured. An error was recorded when the participant selected an item that was different from the target. The number of off-road glances and their duration were recorded. The independent variable was the combination of input and output locations and the dependent variables were: lane deviation, target selection time, target selection accuracy, and the number and duration of glances away from the road. Participants were given a NASA-TLX workload questionnaire upon completion of each condition. The experiment lasted no more than 90 minutes.

12 participants aged 19 to 38 years $(M=27.08, S D=7.3)$ were recruited from our institution. All held a valid driving license and were right-handed. Each participant received $£ 10$ for taking part. 
The study was approved by our institutional ethics committee (Application \#300180296).

\subsection{Apparatus}

The main task consisted of driving a car in a simulated environment on a three-lane motorway. The goal was to drive as straight as possible in the middle lane of a straight road. The vehicle's speed was constant and set to $120 \mathrm{~km} / \mathrm{h}$. The driving task lasted until the participants completed the secondary task.

The secondary task consisted of selecting an item in a scrolling list. The visible area of the list was limited to 12 items and the software included an automatic dwell selection mechanism triggered after 3 seconds of inactivity. This kept input as simple as possible and avoided unnecessary interaction with the system as we were most interested in the location of the tactile input and the perception of the tactile feedback. The list was built using 40 random elements of the periodic table ordered by increasing atomic number to avoid any familiarity that could affect the selection performance.

Scrolling the list was performed by pressing any of the tactile buttons either on the centre console or the steering wheel. The tactile input was implemented through force sensors which prevented any haptic feedback while pressing, allowing us to separate tactile input and output. Pressing the top buttons on either side of the steering wheel made the list scroll upwards, the bottom buttons downwards. On the centre console, pressure on the left button made the list scroll up and on the right made the list scroll down (Figure 1). The tactile stimuli consisted of detents resulting from the transition between each item in the list. Based on Diwishcek and Lisseman's research, haptic detents consisted of an actuation of $200 \mathrm{~ms}$ with a sinusoidal signal at $230 \mathrm{~Hz}$ [7].

The driving simulation was displayed using OpenDS which was customised to allow network communication between the driving simulator and the software running the target selection task [25]. The simulation was displayed on a 100 " projected screen located at 2 meters from the car driving setup. A Logitech G920 gaming steering wheel was used to control the car [18]. No pedals were required as the speed was constant in the study. A custom experimental software was developed and run on a Microsoft Surface as the centre console screen. 3 sets of 2 Peratech QTC SP200-05 force sensors were installed on both sides of the steering wheel and underneath the centre console display as tactile switches [26]. Printed piezoelectric electroactive polymer actuators were installed on each side of the steering wheel for stimulating each hand $[11,30]$. They were actuated using a Texas Instrument DRV2667EVM-CT haptic evaluation module [16]. Because our current prototype does not allow us to drive several printed actuators at once, a haptuator Mark II [17] from Tactile Labs was used to stimulate both driver's hands simultaneously by vibrating the whole steering wheel at once. A Visaton audio exciter EX $60 \mathrm{~S}$ driven by a Visaton Amp 2.2 was used to generate tactile feedback underneath the force sensors on the centre console (Figure 1) [37, 38]. Finally, a Tobii EyeX eye tracker was used to collect participants' eyes movement [36].

\subsection{Procedure}

After the participants sat in the driving simulator, read the information sheet and signed the consent form, they had a training session to experience the input techniques and the tactile feedback at each location. This included its own, separate item list to avoid any learning effects for the main study. Any questions were answered at this time.

For each condition, participants were instructed to keep both hands on the steering wheel when not interacting with the centre console and to use only the specific input that was being evaluated. At the beginning of each condition, the car increased speed to $120 \mathrm{~km} / \mathrm{h}$. After 1000 meters of full speed driving in the centre lane, the first trial started. Each trial began with an audio stimulus to indicate the beginning of the selection task. After the trial start, the participants had to use the button from the relevant input location of the condition to browse the list and select the target which was displayed on the top of the screen. The trial ended as soon as the participant selected an item in the list, which was indicated by a beep. The current selection was highlighted at all times during the trial (Figure 1).

The list order was randomised for each condition. Participants were allowed to rest between each condition. The sound of the driving simulation and of the audio stimuli were delivered through headphones to cover any noise generated by the tactile actuators.

\section{RESULTS}

\subsection{Driving performance}

The smallest lane deviation (root mean square error) was found for $S W L \times S W R(M=0.33, S D=0.10)$ and the largest for $C S \times C S(M=0.41$, $\mathrm{SD}=0.13)$. However, no significant difference was observed.

\subsection{Item selection task performance}

The mean overall success rate for selecting items in the list was 98.6\% ( $\mathrm{SD}=1.29)$. The mean overall selection duration was 12.9 seconds $(\mathrm{SD}=1.5 \mathrm{~s})$. Due to the high selection accuracy across conditions, no further analysis was undertaken.

The mean selection duration was shortest for $S W L \times S W B(M=10.87 \mathrm{~s}$, $\mathrm{SD}=4.54 \mathrm{~s})$ and longest for $C S \times S W L(M=15.68 \mathrm{~s}, \mathrm{SD}=5.20 \mathrm{~s})$. The results are summarised in Figure 2. To accommodate the nonparametric nature of the data distribution, an ART was performed before further analysis [40]. An ANOVA on the transformed data revealed a significant main effect $\left(\mathrm{F}_{11,110}=7.16, \mathrm{p}<.0001\right)$.

Post hoc Tukey's HSD pairwise comparisons found $C S \times C S$ significantly slower than $S W R \times S W L, S W L \times S W B$ and $S W R \times S W R$ (all $\mathrm{t}(110)<4.50, \mathrm{p}<.05) . C S \times C S W L$ was found significantly slower than $S W L \times S W B, S W R \times S W L, S W R \times S W R($ all $\mathrm{t}(110)<4.79, \mathrm{p}<.01)$. This was also the case for $C S \times S W L$ and $S W L \times S W B, S W L \times S W L, S W R \times N o n e$, $S W R \times S W B, S W R \times S W L$ and $S W R \times S W R$ (all $\mathrm{t}(110)<6.30, \mathrm{p}<.05) . S W L \times N o n e$ was likewise significantly slower than $S W L \times S W B$ and $S W R \times S W R$ (all $\mathrm{t}(110)<3.97, \mathrm{p}<.05)$. Finally, $C S \times$ None was found significantly slower than $S W L \times S W B(\mathrm{t}(110)=3.38, \mathrm{p}<.05)$.

\subsection{Glance behaviour}

To measure distraction while participants were driving, we quantified the number of off-road glances and their duration. We established the area of interest containing the road by clustering the participants' fixations from driving with no secondary task [33]. For each fixation from data collected during all the other conditions, 


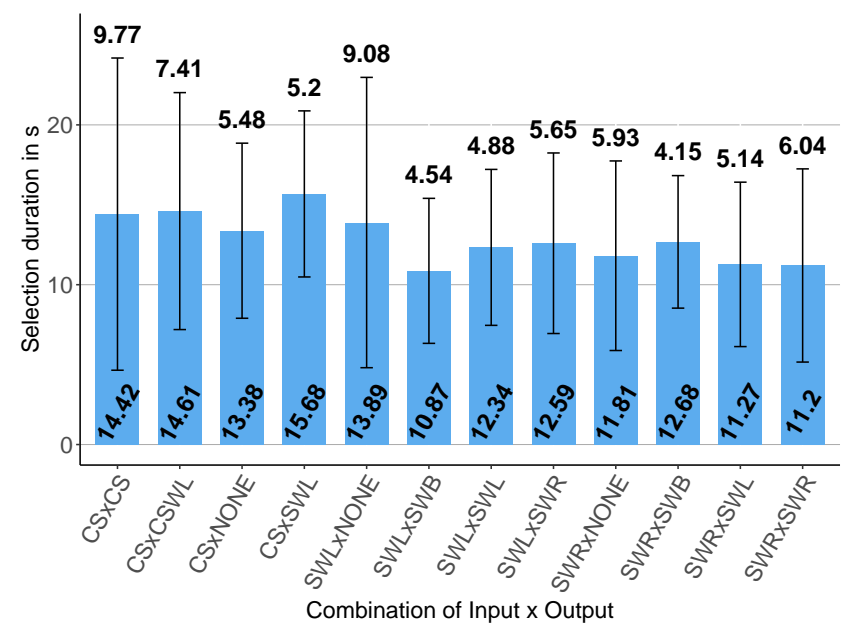

Figure 2: Average item selection duration in seconds. Error bars denote the standard deviation.

we assessed whether they were included in the area of interest. If not, they were identified as glances off the road.

Across all conditions, the average total duration of off-road glances was $10.42 \mathrm{~s}(\mathrm{SD}=1.54 \mathrm{~s})$, the average glance count was 169.23 $(\mathrm{SD}=27.4)$ and the average duration of a single off-road glance was $61 \mathrm{~ms}(\mathrm{SD}=2.7 \mathrm{~ms})$. The results are summarised in Table 2 .

To accommodate the non-parametric nature of the data distribution, an ART was performed before further analysis. The ANOVAs on the transformed data revealed a significant main effect of glance count $\left(\mathrm{F}_{8,80}=4.86, \mathrm{p}<.0001\right)$ and glance duration $\left(\mathrm{F}_{8,80}=2.87, \mathrm{p}<.01\right)$ but no effect was found for the average duration of a single glance. Tukey HSD pairwise comparisons found significantly more off-road glances for $C S \times C S W L$ than $S W L \times S W B, S W L \times S W L$ and $S W R \times S W L$ (all $\mathrm{t}(80)<=3.69, \mathrm{p}<.05)$. There were significantly more off-road glances for $C S \times S W L$ than for $S W L \times S W B, S W L \times S W L$ and $S W R \times S W L$ (all $\mathrm{t}(80)<=3.91, \mathrm{p}<.05)$. However, pairwise comparisons did not find any significant difference for glance duration.

Table 2: Off-road glances total average duration, off-road single glance average duration and off-road glances count. Durations are expressed in seconds and SD values are indicated between parentheses.

\begin{tabular}{llll}
\hline Combination & Total avg. & Count & Single avg. \\
\hline$C S \times C S$ & $12.63(7.05)$ & $181.45(62.75)$ & $0.060(0.016)$ \\
$C S \times C S W L$ & $11.98(4.99)$ & $203.63(89.70)$ & $0.060(0.011)$ \\
$C S \times S W L$ & $12.33(6.52)$ & $219.45(113.84)$ & $0.056(0.007)$ \\
$S W L \times S W B$ & $9.48(6.41)$ & $149.82(90.13)$ & $0.0620 .012)$ \\
$S W L \times S W L$ & $8.58(4.12)$ & $140.73(76.45)$ & $0.063(0.010)$ \\
$S W L \times S W R$ & $9.52(5.13)$ & $155.10(74.67)$ & $0.061(0.009)$ \\
$S W R \times S W B$ & $9.95(5.84)$ & $159.54(83.42)$ & $0.061(0.010)$ \\
$S W R \times S W L$ & $8.73(4.69)$ & $144.73(79.99)$ & $0.061(0.014)$ \\
$S W R \times S W R$ & $10.57(6.39)$ & $168.64(101.49)$ & $0.063(0.012)$ \\
\hline
\end{tabular}

\subsection{Subjective preferences}

The results of the NASA-TLX questionnaire completed after each condition are summarised in Figure 3. An ANOVA on the ART data showed a significant effect for the overall workload $\left(\mathrm{F}_{11,121}=3.34\right.$, $\mathrm{p}<.0001)$, the mental $\left(\mathrm{F}_{11,121}=2.81, \mathrm{p}<.01\right)$ and the physical demand $\left(\mathrm{F}_{11,121}=3.72, \mathrm{p}<.001\right)$, and the perceived effort $\left(\mathrm{F}_{11,121}=2.79, \mathrm{p}<.01\right)$.

Post hoc Tukey HSD pairwise comparisons showed that the average total workload for $C S \times$ None was significantly higher than for $S W L \times S W L((\mathrm{t}(121)=3.76, \mathrm{p}<.05))$ and for $C S \times C S W L$ significantly higher than for $S W L \times S W L(\mathrm{t}(121)=3.35, \mathrm{p}<.05)$. The comparisons also showed $C S \times S W L$ to have significantly higher mental demand than $S W L \times S W R(\mathrm{t}(121)=3.33, \mathrm{p}<.05)$ and $C S \times$ None significantly higher mental demand than $S W L \times S W R$ (all $\mathrm{t}(121)=3.58, \mathrm{p}<.05)$. $C S \times C S W L$ had significantly more physical demand than $S W L \times S W B$, $S W L \times S W L, S W R \times$ None and $S W R \times S W B$ (all $\mathrm{t}(121)<=3.56, \mathrm{p}<.05)$. Finally, $C S \times$ None required more effort than $S W R \times S W B(\mathrm{t}(121)=3.60$, $\mathrm{p}<.05)$.

Data from the post study questionnaire highlighted the fact that participants disliked interacting with the switches located on the centre console. For example, P12 stated "I preferred the button on the right-hand side of the steering wheel, but I did not like the button mounted on the centre" and P8 said: "I liked the steering wheel buttons [as they require] less physical effort". The answers to the questionnaire also revealed that participants preferred when the tactile response was delivered on both hands simultaneously or on the hand used to interact with the IVIS: "I liked it when the tactile feedback was on both sides of the steering wheel as it made it easier to know when the button was pressed" (P12), "I prefer the vibration on the whole steering wheel" (P5).

Participants also thought that the tactile feedback delivered on each side of the steering wheel was too weak and therefore sometimes difficult to perceive: "I preferred the entire wheel vibrating... Hand vibrations [were] too weak" (P11), "too weak" (P8).

However, tactile feedback also helped participants to optimise their performance in the target selection task. P6 said: "Yes, it helps to count the items when browsing the list". P7 shared: "I could easily tell if I had pressed the wrong button because I knew the list wasn't scrolling without looking", "Sometimes I had located the target element which was 1-2 steps away and I could navigate to it almost without looking, because of the feedback", "I could estimate roughly my position [in the list] without looking".

\section{DISCUSSION}

The driving performance data did not reveal any significant difference for the combinations of input and output location. The drivers were able to maintain good road position and were not distracted while operating the haptic controls.

In line with $\mathrm{Ng}$ and Brewster's results, the performance data revealed that participants prioritised accuracy of selection over speed, as the task success rate reached over $98 \%$ overall [23]. The fastest selection performance occurred when interacting using the virtual switches located on the steering wheel and under the nondominant (left) hand, while experiencing tactile vibrations on both hands. The worst performance was found for interacting with the switches located on the centre console while experiencing tactile response on the non-dominant (left) hand. From closer analysis, it 


\begin{tabular}{lll|l|l|l} 
& CSxCS & CSxSWL & SWLXSWL & SWRxSWB \\
Combination Input x Ouput & CSxCSWL & SWLXNONE & SWLXSWR & SWRXSWL \\
\cline { 2 - 5 } & CSxNONE & SWLXSWB & SWRxNONE & SWRxSWR
\end{tabular}

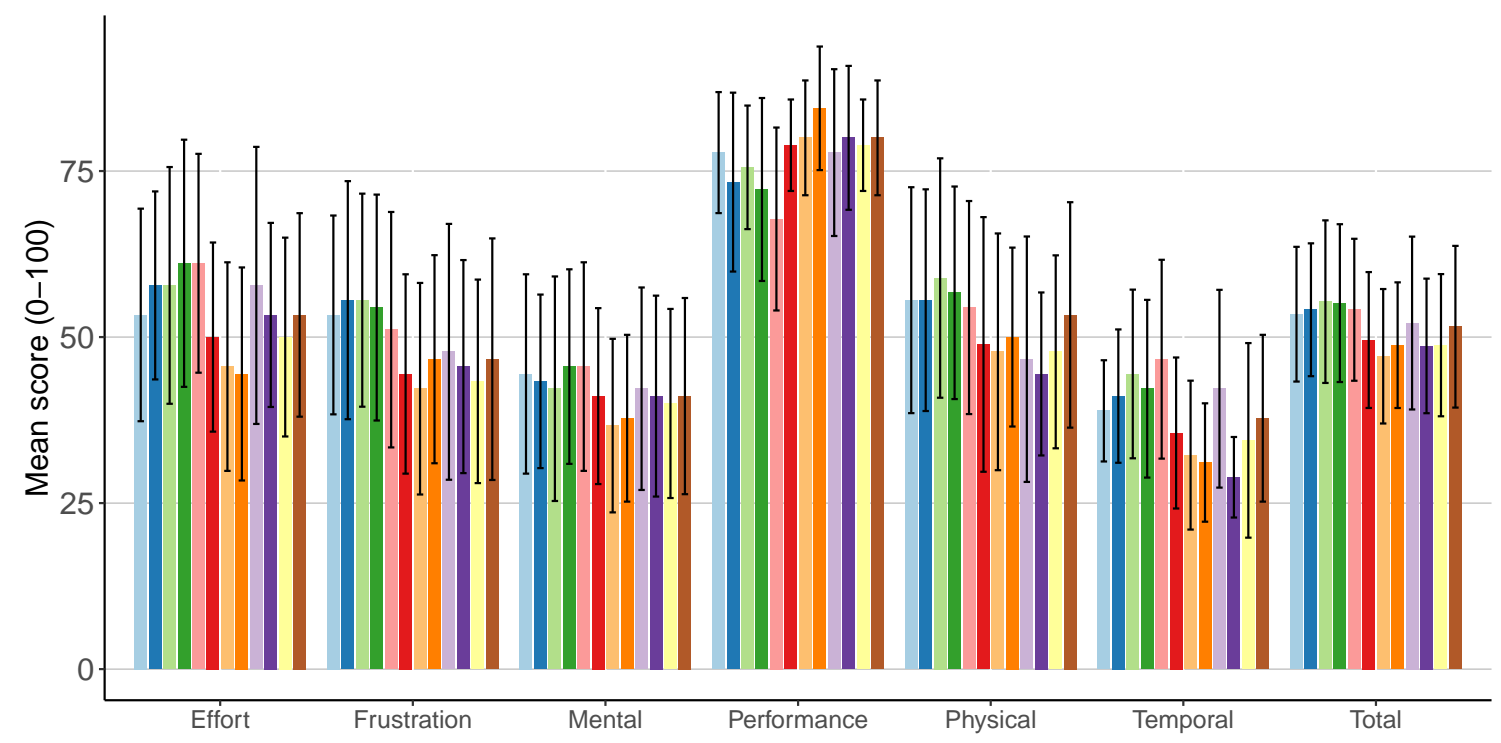

Figure 3: NASA TLX average scores by Input $x$ Output combination. The error bars denote the $95 \%$ confidence interval.

becomes clear that using the switches located on the centre console yielded worse results than interacting with the switches located on the steering wheel, as the performance for $C S \times S W L$ was significantly slower than $S W L \times S W B, S W B \times S W B, S W L \times S W L$ and $S W R \times S W R$, verifying $(\mathrm{H} 2)$. The largest selection duration difference was found between $S W L \times S W B$ and $C S \times S W L$, potentially highlighting the downside of reaching the centre console with the hand and distant tactile feedback. These results are compelling as novel technology allows today's cars to be equipped with enabled tactile feedback displays [3].

Confirming the results on item selection performance, the results from the gaze data revealed that interacting with the switches located on the centre console attracted more glances than interacting with the switches on the steering wheel. The perceived workload results also confirmed the difficulty of interacting with the centre console while driving. Participants generally found that using the switches located on the centre console significantly more mentally and physically demanding, and required more effort than using the switches located on the steering wheel. These results are in line with Döring et al.'s findings [8]. Although previous research has shown that tactile feedback can increase interaction performance with centre consoles, it is worth noting that the average item selection durations of all the centre console combinations with tactile output were longer than any of the steering wheel combinations, and especially the duration for $C S \times$ None being significantly longer than for $S W L \times S W B$ [29]. Because tactile output was also investigated in this comparison for the first time, our findings suggest that, even when tactile output is provided, the centre console remains a poorer location than the steering wheel for interacting with the in-car information system.
Our results show that tactile feedback on the steering wheel improved performance for selecting items in the list as shown by the significantly greater performance of $S W L \times S W B$ and $S W R \times S W R$ over SWL $\times$ None. This only partially validates $\mathrm{H} 1$ as the slowest combination was found when participants interacted with the centre console. Furthermore, the qualitative data confirmed that tactile feedback was helpful for improving participants' performance in selecting the targets in the list. While the tactile output did not show any major difference between the steering wheel and the centre console, it had a significant impact on the steering wheel combinations. Beyond offering tactile feedback on the steering wheel for themes such as driving assistance and warning systems [12], our findings show that it can also serve for supporting non-driving tasks. However, the data did not show any conclusive evidence to pinpoint the best tactile output location on the steering wheel, therefore more research is needed to design the optimal combination to address and integrate these features into a coherent tactile output vocabulary for the steering wheel.

It is worth noting that the data do not allow us to support H3. None of the techniques involving tactile output to the nondominant hand have shown any significant advantage over their dominant-hand counterparts. Furthermore, the shortest item selection durations were found for SWL $\times$ SWB and SWR $\times$ SWR. This was confirmed by participants' preferences going to combinations with the output and the input at the same location, and with the output on both hands. Given these evidences, it is reasonable to believe that offering tactile output to a different location than input is not less cognitively demanding than to the same location or to both hands, ruling out the hypothesis that non-dominant hand stimulation alone may be a better design choice. 
Finally, it should be noted that this study was set for a right-hand traffic setting. While the driving setup configuration may slightly differ, the presented results should remain valid for a left-hand traffic setting as our hypotheses were based on previous research on haptic perception and handedness [34]. It should also be mentionned that our objective was to understand the effect of tactile input and tactile feedback location on drivers' behaviour. For this reason, the use of different types of actuator to generate tactile feedback did not have any effect on the study results as we checked that participants properly felt the tactile feedback at each location of the setup. The study was conducted in a controlled setting which prevented external factors to affect participants' behaviour while they were performing the tasks. However, settings may differ in a realistic driving scenario, as roads may not be straight, and crowded with other vehicles, pedestrians and obstacles. Moreover, command selection mechanisms could be more efficient but also more errorprone than the one in this study. The high cognitive demand of driving in realistic settings might affect the results presented in this paper, nevertheless we believe that these results lay a cornerstone for further research on tactile feedback for new generations of interactive steering wheels.

\section{CONCLUSION}

This paper presents the first study comparing the effects of tactile input and output location (steering wheel and centre console) on non-driving tasks. Our results show that interaction performance on the steering wheel was $30 \%$ higher than on the centre console. The steering wheel is a better location than the centre console for interacting with the in-car information system and input should prevail over output location when designing interaction techniques for drivers. Our results also reveal that performance increased by up to $20 \%$ when participants were provided with tactile feedback. Drivers can benefit from tactile feedback on the steering wheel for non-driving task if it is provided at the same location as the input or to both hands. Our results can be used to improve the design of new generations of interactive tactile steering wheels.

\section{ACKNOWLEDGMENTS}

This research is funded by the European Union's Horizon 2020 research and innovation programme (PRESTIGE, grant agreement ID:\#761112).

\section{REFERENCES}

[1] Rochelle Ackerley, Ida Carlsson, Henric Wester, Håkan Olausson, and Helena Backlund Wasling. 2014. Touch perceptions across skin sites: differences between sensitivity, direction discrimination and pleasantness. Frontiers in Behavioral Neuroscience 8, February (2014), 1-10. https://doi.org/10.3389/fnbeh.2014.00054

[2] Leonardo Angelini, Maurizio Caon, Francesco Carrino, Stefano Carrino, Denis Lalanne, Omar Abou Khaled, and Elena Mugellini. 2013. WheelSense: Enabling Tangible Gestures on the Steering Wheel for In-Car Natural Interaction. In Human-Computer Interaction. Applications and Services, Masaaki Kurosu (Ed.). Springer Berlin Heidelberg, Berlin, Heidelberg, 531-540.

[3] Continental Automotive. $2020 . \quad$ Haptic Feedback Display. https://www.continental-automotive.com/en-gl/PassengerCars/Interior/Display-Systems/Display-Solutions/Haptic-Feedback-Display.

[4] Frank Beruscha, Klaus Augsburg, and Dietrich Manstetten. 2011. Haptic Warning Signals at the Steering Wheel: A Literature Survey Regarding Lane Departure Warning Systems. Haptics-e, the Electronic fournal of Haptic Research 4, 5 (2011) 1-6. http://www.haptics-e.org/Vol\{_\}05/he-v5n1.pdf

[5] Myra Blanco, Wayne J. Biever, John P. Gallagher, and Thomas A. Dingus. 2006. The impact of secondary task cognitive processing demand on driving performance.
Accident Analysis and Prevention 38, 5 (2006), 895-906. https://doi.org/10.1016/j. aap.2006.02.015 arXiv:arXiv:1011.1669v3

[6] Patrizia Di Campli San Vito, Gözel Shakeri, Stephen Brewster, Frank Pollick, Edward Brown, Lee Skrypchuk, and Alexandros Mouzakitis. 2019. Haptic Navigation Cues on the Steering Wheel. In Proceedings of the 2019 CHI Conference on Human Factors in Computing Systems - CHI '19. ACM Press, New York, New York, USA, 1-11. https://doi.org/10.1145/3290605.3300440

[7] Lisa Diwischek and Jason Lisseman. 2015. Tactile Feedback for Virtual Automotive Steering Wheel Switches. In Proceedings of the 7th International Conference on Automotive User Interfaces and Interactive Vehicular Applications (Nottingham, United Kingdom) (AutomotiveUI âÁŹ15). Association for Computing Machinery, New York, NY, USA, 31âĂŞ38. https://doi.org/10.1145/2799250.2799271

[8] Tanja Döring, Dagmar Kern, Paul Marshall, Max Pfeiffer, Johannes Schöning, Volker Gruhn, and Albrecht Schmidt. 2011. Gestural interaction on the steering wheel. In Proceedings of the 2011 annual conference on Human factors in computing systems - CHI '11. ACM Press, New York, New York, USA, 483. https://doi.org/ 10.1145/1978942.1979010

[9] Lucile Dupin, Vincent Hayward, and Mark Wexler. 2015. Direct coupling of haptic signals between hands. Proceedings of the National Academy of Sciences 112, 2 (2015), 619-624. https://doi.org/10.1073/pnas.1419539112

[10] Euan Freeman, Stephen Brewster, and Vuokko Lantz. 2014. Tactile Feedback for Above-Device Gesture Interfaces. In Proceedings of the 16th International Conference on Multimodal Interaction - ICMI '14. ACM Press, New York, New York, USA, 419-426. https://doi.org/10.1145/2663204.2663280

[11] Christian Frisson, Julien Decaudin, Thomas Pietrzak, Alexander Ng, Pauline Poncet, Fabrice Casset, Antoine Latour, and Stephen A. Brewster. 2017. Designing Vibrotactile Widgets with Printed Actuators and Sensors. In Adjunct Publication of the 30th Annual ACM Symposium on User Interface Software and TechnologyUIST '17. ACM Press, New York, New York, USA, 11-13. https://doi.org/10.1145/ 3131785.3131800

[12] Yoren Gaffary and Anatole Lécuyer. 2018. The Use of Haptic and Tactile Information in the Car to Improve Driving Safety: A Review of Current Technologies. Frontiers in ICT 5, March (2018), 1-11. https://doi.org/10.3389/fict.2018.00005

[13] Iván E. González, Jacob O. Wobbrock, Duen Horng Chau, Andrew Faulring, and Brad A. Myers. 2007. Eyes on the Road, Hands on the Wheel: Thumb-Based Interaction Techniques for Input on Steering Wheels. In Proceedings of Graphics Interface 2007 (Montreal, Canada) (GI âĂZO7). Association for Computing Machinery, New York, NY, USA, 95âĂŞ102. https://doi.org/10.1145/1268517.1268535

[14] Camilla Grane and Peter Bengtsson. 2013. Driving performance during visual and haptic menu selection with in-vehicle rotary device. Transportation Research Part F: Traffic Psychology and Behaviour 18 (2013), 123-135. https://doi.org/10. 1016/j.trf.2012.12.011

[15] Jay Henderson, Jeff Avery, Laurent Grisoni, and Edward Lank. 2019. Leveraging Distal Vibrotactile Feedback for Target Acquisition. In Proceedings of the 2019 CHI Conference on Human Factors in Computing Systems - CHI '19. ACM Press, New York, New York, USA, 1-11. https://doi.org/10.1145/3290605.3300715

[16] Texas Instruments. 2020. Texas Instruments EVM for Piezo Haptic Driver with Boost, Digital Front End, and Internal Waveform Memory. http://www.ti.com/tool/DRV2667EVM-CT.

[17] Tactile Labs. 2020. Tactile Labs Haptuator Mark II. http://tactilelabs.com/products/haptics/haptuator-mark-ii-v2/.

[18] Logitech. 2020. Logitech driving force steering wheels. https://www.logitechg.com/en-us/products/driving/driving-force-racingwheel.html.

[19] Christopher McAdam and Stephen Brewster. 2009. Distal Tactile Feedback for Text Entry on Tabletop Computers. In Proceedings of the 23rd British HCI Group Annual Conference on People and Computers: Celebrating People and Technology (Cambridge United Kingdom) (BCS-HCI âĂŹ09). BCS Learning \& Development Ltd., Swindon, GBR, 504âĂŞ511. https://doi.org/10.5555/1671011.1671076

[20] Christopher McAdam and Stephen Brewster. 2011. Using mobile phones to interact with tabletop computers. In Proceedings of the ACM International Conference on Interactive Tabletops and Surfaces - ITS '11. ACM Press, New York, New York, USA, 232. https://doi.org/10.1145/2076354.2076395

[21] Fanxing Meng and Charles Spence. 2015. Tactile warning signals for in-vehicle systems. Accident Analysis \& Prevention 75 (2015), 333-346. https://doi.org/10. 1016/j.aap.2014.12.013

[22] Miyuki Morioka and Michael J. Griffin. 2005. Thresholds for the perception of hand-transmitted vibration: Dependence on contact area and contact location. Somatosensory and Motor Research 22, 4 (2005), 281-297. https://doi.org/10.1080/ 08990220500420400

[23] Alexander Ng and Stephen Brewster. 2017. An Evaluation of Touch and PressureBased Scrolling and Haptic Feedback for In-Car Touchscreens. In Proceedings of the 9th International Conference on Automotive User Interfaces and Interactive Vehicular Applications (Oldenburg, Germany) (AutomotiveUI âĂŹ17). Association for Computing Machinery, New York, NY, USA, 11âĂŞ20. https://doi.org/10. $1145 / 3122986.3122997$ 
[24] Alexander Ng, Stephen A. Brewster, Frank Beruscha, and Wolfgang Krautter. 2017. An Evaluation of Input Controls for In-Car Interactions. In Proceedings of the 2017 CHI Conference on Human Factors in Computing Systems (Denver Colorado, USA) (CHI âÁZ17). Association for Computing Machinery, New York, NY, USA, 2845âĂŞ2852. https://doi.org/10.1145/3025453.3025736

[25] OpenDS. 2020. OPENDS 5.0 - OPEN SOURCE DRIVING SIMULATION. https://opends.dfki.de@misc.

[26] Peratech. 2020. Peratech SP200-05 âĂT̆ Single point touch, Finger electrodes, $5 \mathrm{~mm}$ diameter active area. https://www.peratech.com/sp200-05/.

[27] Sebastiaan M. Petermeijer, David A. Abbink, Mark Mulder, and Joost C.F. De Winter. 2015. The Effect of Haptic Support Systems on Driver Performance: A Literature Survey. IEEE Transactions on Haptics 8, 4 (2015), 467-479. https: //doi.org/10.1109/TOH.2015.2437871

[28] Sebastiaan M. Petermeijer, Joost C. F. de Winter, and Klaus J Bengler. 2016. Vibrotactile Displays: A Survey With a View on Highly Automated Driving. IEEE Transactions on Intelligent Transportation Systems 17, 4 (apr 2016), 897-907. https://doi.org/10.1109/TITS.2015.2494873

[29] Matthew J. Pitts, Gary Burnett, Lee Skrypchuk, Tom Wellings, Alex Attridge, and Mark A. Williams. 2012. Visual-haptic feedback interaction in automotive touchscreens. Displays 33, 1 (2012), 7-16. https://doi.org/10.1016/j.displa.2011 09.002

[30] Pauline Poncet, Fabrice Casset, Antoine Latour, Fabrice Domingues Dos Santos, Sébastien Pawlak, Romain Gwoziecki, Arnaud Devos, Patrick Emery, and Stéphane Fanget. 2017. Static and Dynamic Studies of Electro-Active Polymer Actuators and Integration in a Demonstrator. Actuators 6, 2 (may 2017), 18 https://doi.org/10.3390/act6020018

[31] Hendrik Richter, Sebastian Loehmann, Florian Weinhart, and Andreas Butz. 2012. Comparing Direct and Remote Tactile Feedback on Interactive Surfaces. In Haptics: Perception, Devices, Mobility, and Communication, Poika Isokoski and Jukka Springare (Eds.). Springer Berlin Heidelberg, Berlin, Heidelberg, 301-313.

[32] Hendrik Richter and Alexander Wiethoff. 2011. Augmenting Future In-Vehicle Interactions With Remote Tactile Feedback. In Adjunct Proceedings of the In ternational Conference on Automotive User Interfaces and Interactive Vehicular Applications (AutomotiveUI '11). Association for Computing Machinery, New
York, NY, USA, 162-163. http://www.medien.ifi.lmu.de/pubdb/publications/pub/ richter2011autoUI/richter2011autoUI.pdf

[33] Dario D. Salvucci and Joseph H. Goldberg. 2000. Identifying Fixations and Saccades in Eye-Tracking Protocols. In Proceedings of the 2000 Symposium on Eye Tracking Research and Applications (Palm Beach Gardens, Florida, USA) (ETRA $\hat{a} \breve{A} Z 00$ ). Association for Computing Machinery, New York, NY, USA, 71âĂŞ78. https://doi.org/10.1145/355017.355028

[34] V. Squeri, A. Sciutti, M. Gori, L. Masia, G. Sandini, and J. Konczak. 2012. Two hands, one perception: how bimanual haptic information is combined by the brain. Journal of Neurophysiology 107, 2 (2012), 544-550. https://doi.org/10.1152/ jn.00756.2010

[35] Anthony Talvas, Maud Marchal, and Anatole Lecuyer. 2014. A Survey on Bimanual Haptic Interaction. IEEE Transactions on Haptics 7, 3 (jul 2014), 285-300. https://doi.org/10.1109/TOH.2014.2314456

[36] Tobii. 2020. Tobii Gaming. https://gaming.tobii.com.

[37] Visaton. 2020. Visaton AMP 2.2 LN. https://tinyurl.com/visatonamp.

[38] Visaton. 2020. Visaton structure-borne driver EX $60 \mathrm{~S}-8 \mathrm{Ohm}$ https://tinyurl.com/visatonspeaker.

[39] Steffen Werner. 2014. The Steering Wheel as a Touch Interface. In Proceedings of the 6th International Conference on Automotive User Interfaces and Interactive Vehicular Applications - AutomotiveUI '14. ACM Press, New York, New York, USA, 1-4. https://doi.org/10.1145/2667239.2667299

[40] Jacob O Wobbrock, Leah Findlater, Darren Gergle, and James J Higgins. 2011. The Aligned Rank Transform for nonparametric factorial analyses using only ANOVA procedures. In Conference on Human Factors in Computing Systems Proceedings. ACM Press, New York, New York, USA, 143-146. https://doi.org/10. $1145 / 1978942.1978963$

[41] Katrin Wolf, Anja Naumann, Michael Rohs, and Jörg Müller. 2011. A Taxonomy of Microinteractions: Defining Microgestures Based on Ergonomic and ScenarioDependent Requirements. In Human-Computer Interaction - INTERACT 2011, Pedro Campos, Nicholas Graham, Joaquim Jorge, Nuno Nunes, Philippe Palanque, and Marco Winckler (Eds.). Springer Berlin Heidelberg, Berlin, Heidelberg, 559575 